\title{
The relationships between nasal hyperreactivity, quality of life, and nasal symptoms in patients with perennial allergic rhinitis
}

\author{
Tineke de Graaf-in 't Veld, PhD, a Susanne Koenders, MA, a \\ Ingrid M. Garrelds, PhD, ${ }^{\mathrm{a}, \mathrm{b}}$ and Roy Gerth van Wijk, PhDa \\ Rotterdam, The Netherlands
}

\begin{abstract}
Background: $A$ clinical test that could inform the clinician about the severity of a patient's nasal symptoms and health-related quality of life $(Q O L)$ would be very useful.

Objective: We attempted to determine whether, in patients with perennial allergic rhinitis, nasal challenge with histamine could be used to estimate daily symptoms and QOL.

Methods: Forty-eight patients with perennial allergic rhinitis were challenged with histamine to determine nasal hyperreactivity. Nasal response was monitored by the number of sneezes, the amount of secretion, and a symptom score. Daily nasal symptoms were recorded during the 2 preceding weeks. Patients also completed a rhinitis QOL questionnaire.

Results: Responsiveness to histamine and total daily nasal symptoms were moderately correlated $(\mathrm{r}=0.51, \mathrm{p}=0.001)$. Comparison of total daily nasal symptoms with the overall $Q O L$ score showed a moderate correlation $(\mathrm{r}=0.59, \mathrm{p}<0.001)$. Nasal response to histamine and overall QOL score were also correlated $(\mathrm{r}=0.43, \mathrm{p}=0.002)$. However, overall QOL and daily nasal symptoms could be predicted by wide $95 \%$ confidence intervals only for each decade of nasal responsiveness to histamine (expressed as a composite symptom score).

Conclusion: In patients with perennial allergic rhinitis nasal hyperreactivity as determined by histamine challenge, $Q O L$, and daily nasal symptoms are moderately correlated. Therefore nasal histamine challenge can be used as a tool for estimating the severity of daily nasal symptoms and QOL, although it cannot predict nasal symptoms and QOL very accurately. (J Allergy Clin Immunol 1996;98:508-13.)
\end{abstract}

Key words: Perennial allergic rhinitis, daily symptoms, quality of life, nasal hyperreactivity, histamine challenge

Patients with allergic rhinitis experience sneezing, nasal itchiness, rhinorrhea, and nasal blockage after exposure to the relevant allergen. After repeated exposure to allergen, nasal responsiveness to allergens increases. This phenomenon, known as nasal priming, was first described by Connell ${ }^{1}$

From athe Department of Allergology, University Hospital Rotterdam-Dijkzigt; and bthe Department of Pharmacology, Erasmus University Rotterdam, The Netherlands.

Supported by grant 92.74 of the Dutch Asthma Foundation, Leusden, the Netherlands.

Received for publication Mar. 21, 1995; revised Dec. 28, 1995; accepted for publication Jan. 5, 1996.

Reprint requests: C. de Graaf-in 't Veld, Department of Allergology, University Hospital Dijkzigt, Dr. Molewaterplein 40, 3015 GD Rotterdam, The Netherlands.

Copyright (c) 1996 by Mosby-Year Book, Ine.

$0091-6749 / 96 \$ 5.00+0 \quad \mathbf{1 / 1 / 7 1 7 8 3}$
Abbreviation used

QOL: Quality of life

and was confirmed by others. ${ }^{2,3}$ Exposure to allergen also increases nasal responsiveness to nonspecific stimuli. ${ }^{4}$ This nonspecific nasal hyperreactivity is particularly important in patients with perennial allergic rhinitis, because they are continually exposed to allergen.

Hyperreactivity can be described as a clinical feature characterized by occurrence of symptoms on exposure to daily-life stimuli as estimated by history (clinical hyperreactivity). Determination of nasal hyperreactivity by nasal 
provocation tests with pharmacologic or physical agents assumes a relationship between this form of nasal hyperreactivity and hyperreactivity as estimated by history. Gerth van Wijk et al. ${ }^{5}$ demonstrated that the amount of secretion and the number of sneezes in response to histamine challenge were associated with the clinical hyperreactivity assessed by a hyperreactivity score.

The importance of routine histamine challenges in daily practice is not clear. In a previous study we showed that assessment of nasal airway resistance after histamine challenge is less appropriate in distinguishing patients from healthy subjects in terms of reproducibility and estimation of clinical hyperreactivity compared with assessment of number of sneezes and amount of secretion. ${ }^{6}$ Recently, we demonstrated that reactivity to histamine expressed as area under the curve with a composite symptom score (according to the method of Lebel et al. ${ }^{7}$ ) as response variable is a valid method for detecting treatment effect. ${ }^{8}$ In addition, the method is valid for distinguishing between patients with and without a latephase allergic reaction. ${ }^{9}$ Moreover, this method appears to be reproducible. ${ }^{10}$

Recently, a correlation between daily symptoms and response to histamine challenge was demonstrated in patients with nonallergic nasal hyperreactivity. ${ }^{11} \mathrm{We}$ attempted to determine whether, in patients with perennial allergic rhinitis, nasal hyperreactivity (responsiveness to histamine) corresponds with daily nasal symptoms. In addition to the amount of secretion and the number of sneezes, the symptom score of Lebel et al. ${ }^{7}$ was used to monitor the nasal responsiveness to histamine.

Patients' nasal symptoms also interfere with their day-to-day lives. To measure the influence of nasal symptoms on day-to-day life, rhinitis quality of life (QOL) questionnaires have been developed. ${ }^{12-14}$ Juniper et al. ${ }^{12,13}$ demonstrated that QOL deteriorated after allergen exposure (pollen season) and increased after symptomatic treatment.

We performed this study to determine whether, in patients with perennial allergic rhinitis, the daily nasal symptoms are correlated with $\mathrm{QOL}$ and also whether nasal hyperreactivity, as measured by nasal histamine challenge, is correlated with $\mathrm{QOL}$ scores and nasal symptoms. Furthermore, we attempted to determine whether nasal histamine challenge can be used as a tool to estimate the severity of patients' daily nasal symptoms and health-related QOL.

\section{METHODS Study design}

Each adult patient with perennial allergic rhinitis visiting our outpatient clinic for the first time completed a nasal symptom diary during the 2 weeks preceding the follow-up visit. At this second visit, patients completed a rhinitis QOL questionnaire and underwent a nasal histamine challenge.

\section{Subjects}

Forty-eight patients (23 men and 25 women; mean age, $30 \pm 10$ years) with a history of perennial rhinitis participated in this study. They had to meet the following criteria: perennial rhinitis for at least 1 year; positive intradermal skin test response to at least one perennial allergen (house dust mites and/or molds and/or animal dander; ALK Benelux, Groningen, The Netherlands) and exposure to the allergen(s) involved. Of the patients included, 38 were allergic to house dust mites, seven were allergic to house dust mites and pets (and had a pet at home), two were allergic to their pets only, and one was allergic to molds. Patients with a concomitant allergy to pollen were only included outside the pollen season. During the study they were not permitted to use any medication affecting nasal symptoms, apart from Acrivastine, $8 \mathrm{mg}$, (as rescue medication). Acrivastine had to be withdrawn 3 days before the histamine challenge. Patients with a nasal infection were excluded.

\section{Nasal symptom diary}

Patients recorded their nasal symptoms for 2 weeks on a modified diary card according to the symptom score system of Norman et al. ${ }^{15}$ Sneezes/itching nose, nasal blockage, and rhinorrhea were scored according to their daily duration $(0=$ no symptoms, $1=$ symptoms $<1$ hour, 2 = symptoms between 1 and 4 hours, $3=$ symptoms $>4$ hours). The sum of the individual symptoms during the 2 weeks and the total nasal symptom score (sneezing/itchiness + rhinorrhea + nasal blockage) during these 2 weeks were used for statistical analysis.

\section{Rhinitis QOL questionnaire}

We translated the rhinitis QOL questionnaire developed by Juniper et al. ${ }^{13}$ to Dutch. Patients had to score 25 items subdivided into the following domains: sleep ( $n$ $=3$ ), nasal symptoms $(n=5)$, practical problems $(n=$ $3)$, emotions $(n=4)$, activities $(n=3)$, and nonrhinitis symptoms $(n=7)$. In each item, they were asked how much they were troubled as a result of their nasal symptoms during the previous week. The score of these items ranged from 0 (not troubled) to 6 points (extremely troubled). In case of the items concerning emotions, patients were asked how often they were troubled, in each of the items, as a result of their nasal symptoms. The score also ranged from 0 (never troubled) to 6 points (constantly troubled). In the analysis the mean within-patient score of each QOL domain was used 
TABLE I. Nasal hyperreactivity versus daily nasal symptoms $(n=40)$

\begin{tabular}{lccc}
\hline & \multicolumn{3}{c}{ Nasal hyperreactivity } \\
\cline { 2 - 4 } \multicolumn{1}{c}{$\begin{array}{c}\text { Daily nasal } \\
\text { symptoms }\end{array}$} & $\begin{array}{c}\text { Sneezes } \\
(\boldsymbol{r})\end{array}$ & $\begin{array}{c}\text { Secretion } \\
(\boldsymbol{r})\end{array}$ & $\begin{array}{c}\text { Symptom score } \\
(\boldsymbol{r})\end{array}$ \\
\hline Itchiness/sneezing & 0.00 & $\mathbf{0 . 2 2}$ & $0.49^{*}$ \\
Secretion & 0.04 & $0.41^{*}$ & $0.39^{*}$ \\
Congestion & 0.14 & 0.05 & $0.31^{*}$ \\
Total symptoms & 0.10 & 0.24 & $0.51^{*}$ \\
\hline
\end{tabular}

$r$, Spearman's correlation coefficient.

* $p<0.05$.

(these mean domain scores were measured by calculating the mean of the items within each domain). In addition, the total score of the means of the six domains was used to calculate the overall QOL score.

\section{Nasal histamine challenge}

Challenges were performed according to the methods described by Gerth van Wijk et al..$^{5} 6$ Patients waited for half an hour before the test to allow the nasal mucosa time to acclimatize. Nasal challenge with histamine phosphate $(0.25,0.5,1.0,2.0$, and $4.0 \mathrm{mg} / \mathrm{ml})$ was performed at 5-minute intervals after challenge with phosphate-buffered saline. The phosphate-buffered saline and the increasing doses of histamine were sprayed into the nostrils with a nasal pump spray, delivering a fixed dose of $0.125 \mathrm{ml}$ of solution. After each challenge, the symptom score ${ }^{7}$ was recorded, sneezes were counted, and anterior rhinorrhea was measured by collecting nasal secretions in a syringe-equipped funnel. ${ }^{16}$ The areas under the curve of histamine dose response were used in the statistical analysis.

\section{Symptom score}

The symptom score according to Lebel et al. ${ }^{7}$ was used: three to four sneezes $=1$ point, five or more sneezes $=3$ points, anterior rhinorrhea $=1$ point, posterior rhinorrhea $=1$ point, difficult nasal breathing $=1$ point, one nostril blocked $=2$ points, both nostrils blocked $=3$ points, pruritus of the nose $=1$ point, pruritus of the palate or ear $=1$ point, and conjunctivitis $=1$ point. Total score ranged from 0 to 11 points.

\section{Statistics}

Forty patients completed their nasal symptom diary correctly; in comparing nasal symptoms with QOL scores and nasal hyperreactivity, we used data of these patients only ( $n=40$ ). When the correlation between QOL scores and nasal hyperreactivity was tested, data from all subjects could be used $(n=48)$.

Because not all variables were normally distributed, relationships between the variables were tested by Spearman's rank correlation. A two-tailed $p$ value of less than 0.05 was considered significant. Furthermore, the
TABLE II. QOL versus nasal hyperreactivity $(n=48)$

\begin{tabular}{|c|c|c|c|}
\hline \multirow[t]{2}{*}{ QOL domain } & \multicolumn{3}{|c|}{ Nasal hyperreactivity } \\
\hline & $\begin{array}{c}\text { Sneezes } \\
(r)\end{array}$ & $\begin{array}{c}\text { Secretion } \\
(r)\end{array}$ & $\begin{array}{c}\text { Symptom score } \\
\text { (n) }\end{array}$ \\
\hline Sleep & 0.15 & $0.36^{*}$ & 0.27 \\
\hline Nonrhinitis symptoms & 0.28 & $0.34^{*}$ & $0.29^{*}$ \\
\hline Practical problems & 0.10 & 0.25 & $0.34^{*}$ \\
\hline Nasal symptoms & 0.26 & 0.18 & $0.47^{*}$ \\
\hline Activities & $0.37^{*}$ & 0.21 & $0.32^{*}$ \\
\hline Emotions & 0.07 & 0.16 & 0.24 \\
\hline Overall QOL & $0.31^{*}$ & $0.32 *$ & $0.43^{*}$ \\
\hline
\end{tabular}

$r$, Spearman's correlation coefficient.

${ }^{*} p<0.05$.

mean predicted values and $95 \%$ confidence intervals of the overall QOL score and total nasal symptoms for each decade of nasal response to histamine challenge were determined by multiple regression analysis (this method could be used because these three variables were normally distributed).

\section{RESULTS}

Table I shows correlation coefficients of daily nasal symptoms compared with nasal response to histamine. Histamine responsiveness is expressed as the area under the curve of the amount of secretion, the number of sneezes, and the symptom score of Lebel et al. ${ }^{7}$ after nasal histamine challenge. Moderate but statistically significant correlations were found between the daily symptoms and histamine responsiveness, expressed as symptom score of Lebel et al. ${ }^{7}$ The secretory response to histamine is moderately correlated with daily secretion only. When histamine responsiveness was expressed as the number of sneezes, no correlations were found.

Table II shows the correlation coefficients of nasal response to histamine and QOL domains. The symptom score of Lebel et al. ${ }^{7}$ correlates moderately with most QOL domains, with the exceptions of sleep and emotions. However, the correlations with sleep and emotions tended to be significant $(r=0.27, p=0.067$ and $r=0.24, p=$ 0.098 , respectively). The number of sneezes is correlated with the activities and with overall QOL score only. Secretory response to histamine is correlated with sleep, nonrhinitis symptoms, and the overall QOL score.

When total daily nasal symptoms are compared with the QOL domains, moderate but statistically significant correlations are shown for all QOL domains and the overall QOL score (Table III). Of 
TABLE III. QOL versus daily nasal symptoms $(n=40)$

\begin{tabular}{lcccc}
\hline & \multicolumn{4}{c}{ Daily nasal symptoms } \\
\cline { 2 - 5 } OoL domain & Itchiness/sneezing $(r)$ & Secretion $(r)$ & Congestion $(r)$ & Total $(r)$ \\
\hline Sleep & $0.33^{*}$ & 0.25 & 0.29 & $0.35^{*}$ \\
Nonrhinitis symptoms & $0.37^{*}$ & 0.19 & 0.29 & $0.36^{*}$ \\
Practical problems & $0.57^{*}$ & $0.37^{*}$ & $0.39^{*}$ & $0.56^{*}$ \\
Nasal symptoms & $0.61^{*}$ & $0.43^{*}$ & 0.23 & $0.54^{*}$ \\
Activities & $0.46^{*}$ & 0.14 & $0.52^{*}$ & $0.45^{*}$ \\
Emotions & 0.29 & 0.19 & $0.36^{*}$ & $0.36^{*}$ \\
Overall QOL & $0.59^{*}$ & $0.36^{*}$ & $0.47^{*}$ & $0.59^{*}$ \\
\hline
\end{tabular}

$r$, Spearman's correlation coefficient.

${ }^{*} p<0.05$.

the individual symptoms, itchiness/sneezing is correlated with five of the six QOL domains and the overall QOL, congestion with three domains and the overall QOL, and secretion with two domains and the overall QOL only. Because of the withinpatient variability of the daily symptoms, the $p$ values might be too optimistic.

When daily acrivastine intake was added to the total score of the diary, as Norman et al. ${ }^{15}$ did, the correlation coefficients of total daily symptoms with hyperreactivity were 0.05 (sneezes), 0.26 (secretion), and 0.51 (symptom score) compared with $0.10,0.24$, and 0.51 without addition of acrivastine. Correlations of total daily symptoms with QOL domains ranged from 0.37 to 0.60 compared with 0.35 to 0.59 with or without acrivastine, respectively. So acrivastine intake did not change the correlation coefficients. In Fig. 1 the association between overall QOL and total daily symptoms is shown $(r=0.59, p<0.001)$.

In Table IV predicted values of and $95 \%$ confidence intervals for each decade of nasal response to histamine are demonstrated. Nasal response to histamine explained $21 \%$ of the variance of nasal symptoms and $21 \%$ of the variance of QOL.

\section{DISCUSSION}

We attempted to determine whether routine nasal histamine challenges in patients with perennial allergic rhinitis can be used to give an impression of the severity of patients' daily nasal symptoms. Nasal response to histamine, if expressed as the symptom score of Lebel et al., ${ }^{7}$ correlated moderately with sneezes/itchiness, rhinorrhea, congestion, and total nasal symptoms. Secretory response to histamine correlated only weakly with nasal symptoms, whereas the number of sneezes in response to histamine challenge was not correlated with daily nasal symptoms at all. This is in contrast to a previous study by Gerth van Wijk et al., ${ }^{5}$ who demonstrated that histamine-induced sneezes and secretion correlated well with daily nasal symptoms. However, the diary they used contained, besides the symptom score system of Norman et al., ${ }^{15}$ specific questions about nasal hyperreactivity, so the two diaries are not completely comparable. This might suggest that routine histamine challenges in daily practice, if monitored by the symptom score of Lebel et al., ${ }^{7}$ give a better impression of the severity of patients' daily symptoms. The correlations, however, were not very strong, and daily nasal symptoms could be predicted by wide $95 \%$ confidence intervals only. One clinical test cannot be expected to exactly predict daily nasal symptoms. However, it might be used in addition to a carefully taken history to make a better estimation of the severity of patients' symptoms.

Patients with rhinitis may have nasal symptoms that adversely affect their day-to-day lives. We used the rhinitis QOL questionnaire developed by Juniper et al. ${ }^{13}$ to measure the effect of rhinitis on day-to-day life. Juniper et al. ${ }^{12,13}$ have validated this questionnaire and have shown that it is a useful instrument in clinical trials to detect withinsubject changes over time (treatment effect, allergen exposure). We did not study changes in QOL, but we attempted to determine whether QOL scores are correlated with the daily nasal symptoms and nasal response to histamine.

Malo et al. ${ }^{17}$ demonstrated that in patients with asthma, QOL and bronchial hyperreactivity were correlated. Because the relationship between nasal hyperreactivity and QOL has not been studied yet, we investigated whether in patients with perennial allergic rhinitis, such a relationship could also be demonstrated. Nasal hyperreactivity was determined by nasal response to histamine. When his- 


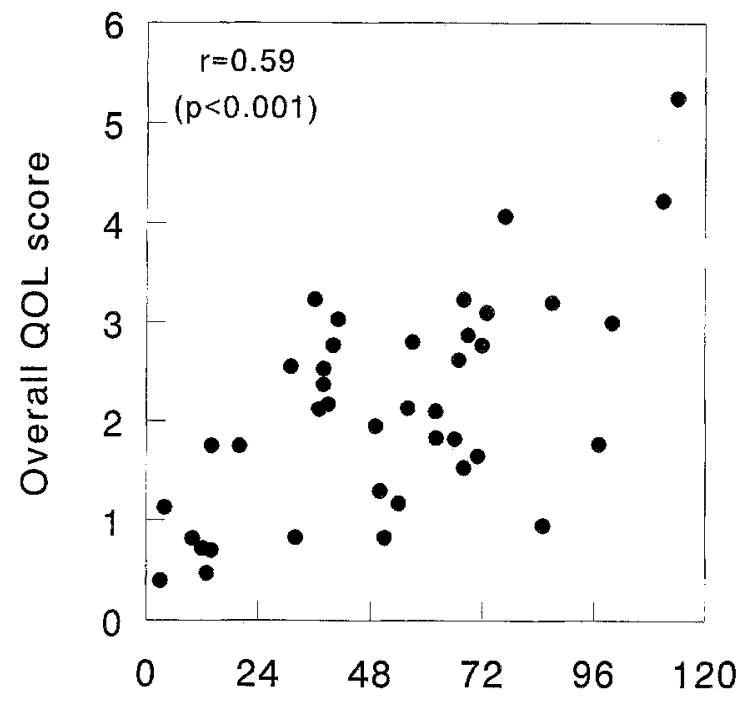

\section{Total daily nasal symptoms}

FIG. 1. Overall $\mathrm{QOL}$ score plotted against total daily nasal symptoms recorded during 2 weeks $(n=40)$.

tamine responsiveness was expressed as the symptom score, significant correlations were found with all QOL domains, with the exceptions of emotions and sleep. The correlation coefficients found in our rhinitis study were moderate $(0.24<r<0.47)$; however, they were equal to or higher than those in asthma studies. Correlation coefficients between bronchial responsiveness to methacholine found by Malo et al. ${ }^{17}$ ranged from -0.1 to -0.24 . Marks et al. ${ }^{18}$ studied the relationship between changes in bronchial hyperreactivity and changes in QOL; correlation coefficients ranged from 0.14 to 0.47 .

The number of sneezes and the amount of secretion in response to histamine challenge correlated only slightly with some of the QOL domains. So when nasal hyperreactivity is compared with QOL, nasal hyperreactivity expressed as the symptom score of Lebel et al. ${ }^{7}$ is a better variable than the number of sneezes and/or the amount of secretion.

When QOL was compared with nasal symptom diaries, all QOL scores were correlated with total nasal symptoms, indicating that in patients with more severe rhinitis symptoms, QOL is more impaired. The moderate correlations between nasal symptoms and the nasal symptom domain of QOL might be due to the individual perception of the degree of impairment caused by the nasal symptoms. Some patients might be impaired by only a few nasal symptoms, whereas others might be less impaired by more
TABLE IV. Predicted nasal symptoms $(n=$ $40)$ and $Q O L(n=48)$ for each decade of the hyperreactivity range

\begin{tabular}{|c|c|c|c|c|}
\hline \multirow{2}{*}{$\begin{array}{l}\text { Hyperreactivity } \\
\text { symptom } \\
\text { score }\end{array}$} & \multicolumn{2}{|c|}{$\begin{array}{l}\text { Predicted nasal } \\
\text { symptom score }\end{array}$} & \multicolumn{2}{|c|}{$\begin{array}{l}\text { Predicted } \\
\text { OOL score }\end{array}$} \\
\hline & Mean & $95 \% \mathrm{Cl}$ & Mean & $95 \% \mathrm{Cl}$ \\
\hline 10 & 34.98 & $0.00-88.34$ & 1.43 & $0.00-3.44$ \\
\hline 20 & 48.46 & $0.00-100.79$ & 1.89 & $0.00-3.88$ \\
\hline 30 & 61.94 & $0.31-114.57$ & 2.36 & $0.37-4.35$ \\
\hline 40 & 75.42 & $21.19-129.65$ & 2.81 & $0.79-4.85$ \\
\hline 50 & 88.90 & $31.88-145.93$ & 3.29 & $1.18-5.39$ \\
\hline
\end{tabular}

CI, Confidence interval.

severe nasal symptoms. The correlation coefficients $(0.35$ to 0.59$)$ we found are about in the same range as those found by Juniper and Guyatt ${ }^{12}$ ( 0.31 to 0.59$)$; however, the results are not totally comparable, because Juniper and Guyatt compared changes in nasal symptoms with changes in QOL.

When QOL scores were compared with the individual nasal symptoms, a better correlation was seen with sneezes/itchiness than with rhinorrhea and congestion, so that it seems that sneezing/ itchiness causes the most impairment of day-to-day life. Perhaps patients become used to having stuffy noses and blowing their noses, but sneezing and nasal itchiness are hard to ignore and more difficult to accept. Because the Dutch version of the rhinitis QOL questionnaire developed and validated by Juniper et al. ${ }^{13}$ was correlated with daily nasal symptoms, it can also be used in the Netherlands as a valid tool to measure rhinitis QOL.

When acrivastine intake was added to the total nasal symptoms, as Norman et al. ${ }^{15}$ did, the correlations between total nasal symptoms and QOL or responsiveness to histamine challenge did not change. So it seems to make no difference whether acrivastine intake is included or not. Norman et al. ${ }^{15}$ also found that using the symptom diary to interpret the clinical effectiveness of immunotherapy made no difference, whether the use of antihistamines was included or not.

In conclusion, in patients with perennial allergic rhinitis nasal hyperreactivity, QOL, and daily nasal symptoms are moderately correlated. Therefore routine histamine challenge, if expressed by a composite symptom score, can be used as tool to obtain an impression of daily nasal symptoms and rhinitis QOL, although it cannot predict nasal symptoms and QOL very accurately. 
We thank Dr. Elisabeth Juniper for her comments on the manuscript and Dr. Paul G. H. Mulder for his assistance with the statistical analysis.

\section{REFERENCES}

1. Connell JT. Quantitative intranasal pollen challenge. II. Effect of daily pollen challenge, environmental pollen exposure and placebo challenge on the nasal membrane. J Allergy 1968;41:123-39.

2. Bacon JR, McLean JA, Mathews KP, Banas JM. Priming of the nasal mucosa by ragweed extract or by an irritant (ammonia). J Allergy Clin Immunol 1981;67:111-6.

3. Grønborg H, Borum P, Mygind N. Histamine and methacholine do not increase nasal reactivity. Clin Allergy 1986; 16:597-602.

4. Andersson M, Andersson P, Pipkorn U. Allergen-induced specific and non-specific nasal reactions. Acta Otolaryngol 1989;107:270-7.

5. Gerth van Wijk R, Mulder PGH, Dieges PH. Nasal provocation with histamine in allergic rhinitis patients: clinical significance and reproducibility. Clin Exp Allergy 1989;19: 293-8.

6. Gerth van Wijk R, Dieges PH. Comparison of nasal responsiveness to histamine, methacholine and phentolamine in allergic rhinitis patients and controls. Clin Allergy 1987; 17:563-70.

7. Lebel B, Bousquet J, Morel A, Chanal I, Godard P, Michel FB. Correlation between symptoms and the threshold for release of mediators in nasal secretions during nasal challenge with grass-pollen grains. J Allergy Clin Immunol 1988;82:869-77.

8. De Graaf-in 't Veld C, Garrelds IM, Jansen APH, et al. Effect of fluticasone propionate on the immediate and late allergic reaction and nasal hyperreactivity in patients with a house dust mite allergy. Clin Exp Allergy 1995;25:966-73.
9. Gerth van Wijk R, de Graaf-in 't Veld C, Garrelds IM, van Toorenenbergen AW, Zijlstra FJ. Relationship between nasal responsiveness to allergen and histamine in patients with perennial rhinitis [abstract]. J Allergy Clin Immunol $1994 ; 93 ; 273$.

10. De Graaf-in 't Veld C, Garrelds IM, Koenders S, Gerth van Wijk R. Relationship between nasal hyperreactivity, mediators and eosinophils in patients with perennial allergic rhinitis and controls. Clin Exp Allergy. In press.

11. Hallén $\mathrm{H}$, Juto JE. Correlation between subjective and objective assessment of nasal hyperreactivity. ORL J Otorhinolaryngol Relat Spec 1994;56:51-4.

12. Juniper EF, Guyatt GH. Development and testing of a new measure of health status for clinical trials in rhinoconjunctivitis. Clin Exp Allergy 1991;21:77-83.

13. Juniper EF, Guyatt GH, Andersson B, Ferrie PJ. Comparison of powder and aerosolized budesonide in perennial rhinitis: validation of rhinitis quality of life questionnaire. Ann Allergy 1993;70:225-30.

14. Bousquet $\mathbf{J}$, Bullinger $\mathrm{M}$, Fayol $\mathrm{C}$, Marquis P, Valentin B, Burtin B. Assessment of quality of life in patients with perennial allergic rhinitis with the French version of the SF-36 Health Status Questionnaire. J Allergy Clin Immunol 1994;94:182-8.

15. Norman PS, Winkenwerder WL, Lichtenstein LM. Trials of alum-precipitated pollen extracts in the treatment of hay fever. J Allergy Clin Immunol 1972;50:31-44.

16. Borum P. Nasal methacholine challenge. J Allergy Clin Immunol 1979;63:253-7.

17. Malo JL, Boulet LP, Dewitte JD, et al. Quality of life of subjects with occupational asthma. J Allergy Clin Immunol 1993;91:1121-7.

18. Marks GB, Dunn SM, Woolcock AJ. An evaluation of an asthma quality of life questionnaire as a measure of change in adults with asthma. J Clin Exp Allergy 1993; 46:1103-11. 